\title{
Comparing Israeli and Palestinian polio vaccination policies and the challenges of silent entry of wild poliovirus in 2013-14: a 'natural experiment'
}

\author{
Antoine Flahault • Walter Orenstein · Julie Garon • \\ Olen Kew $\cdot$ Joan Bickford $\cdot$ Theodore Tulchinsky
}

Published online: 4 July 2015

(C) Swiss School of Public Health 2015

Eradication of poliomyelitis has been a long time global challenge and is currently reaching the final end stages (Moturi et al. 2014). The potential for reappearance of both wild poliovirus (WPV) and other vaccine-related polioviruses has impacted policy development and influenced strategies implemented worldwide (World Health Organization 2013; Mundel and Orenstein 2013).

This commentary views the use of a combined oral polio vaccine (OPV) and inactivated polio vaccine (IPV) schedule in comparison to IPV-alone polio immunization

This commentary is part of the special issue "Driving the Best Science to Meet Global Health Challenges" edited on the occasion of the 9th European Congress on Tropical Medicine and International Health 2015.

\section{A. Flahault $(\bowtie)$}

Faculte de Medecine, Universite de Geneve, Geneva,

Switzerland

e-mail: antoine.flahault@unige.ch

\section{A. Flahault}

Centre Virchow-Villermé, Descartes School of Medicine,

Sorbonne Paris, Cité, Paris, France

W. Orenstein · J. Garon

Division of Infectious Diseases, Emory University School of Medicine, Atlanta, GA, USA

O. Kew

National Center for Immunization and Respiratory Diseases, Centers for Disease Control and Prevention, Atlanta, GA, USA

J. Bickford

Manitoba Health, Winnipeg, Manitoba, Canada

T. Tulchinsky

Braun School of Public Health, Hebrew University-Hadassah, Ein Karem, Jerusalem, Israel programs in limiting the spread of imported WPV. In 2013, type 1 wild poliovirus (WPV1) entered highly immunized Israel and Palestinian Territories from Egypt and circulated for more than a year (Anis et al. 2013; Manor et al. 2014).

During the 1970s, Gaza and the West Bank experienced high levels of clinical poliomyelitis with many cases occurring among children who had received multiple doses of OPV. In the early 1980s, polio was eliminated in both areas using a combination of OPV and IPV (Goldblum et al. 1994).

Israel used OPV nationwide, except for in two districts in which an IPV-only schedule was used. Following a 1988 outbreak of poliomyelitis in one of these areas (Hadera) in which 15 cases of polio occurred, Israel adopted a combined OPV/IPV schedule. No further cases were found and a routine sewage monitoring system for polioviruses was initiated in Israel, the West Bank and Gaza (Slater et al. 1990).

In 2004, Israel adopted an IPV-only policy in keeping with practices adopted in European and North American countries. Having achieved polio-free status, these countries sought to prevent vaccine-associated poliomyelitis (VAPP), a more pressing national priority at that time than prevention of WPV transmission, which was no longer endemic.

In 2013, routine monitoring tests showed WPV1 in sewage in southern and later central Israel. Increased surveillance, developed in consultation with international experts, indicated widespread WPV1 isolates in Southern and Central Israel continuing into early 2014. The positive sewage findings were primarily in Israel, initially among Bedouin communities, but later spreading to mixed communities in Central Israel. A mass campaign of OPV was conducted for children up to age 10 in early 2014. Increased sampling in the West Bank and Gaza showed 
few positives, but the Palestinian Authority conducted a two-stage OPV booster campaign as well.

No cases of poliomyelitis occurred in either Israel or the Palestinian Territories.

This "natural experiment" compared experiences of combined (sequential) use of both IPV and OPV to an IPVonly program in protecting against silent entry and spread of WPV, even into areas with high immunization coverage. Both schedules were found to protect against clinical polio, but the combined OPV/IPV program seemed to induce greater protection against silent spread of imported WPV (Mangal et al. 2013; Tulchinsky et al. 2013).

We believe that this experience supports the following recommendations:

- Sewage monitoring for poliovirus is a necessary tool for detection of poliovirus in the end stages of polio eradication.

- Adding IPV in OPV-only countries will reduce the risk of VAPP, boost intestinal immunity and decrease the potential for silent spread of imported WPV in communities. This policy has already been adopted by the World Health Organization (Weekly Epidemiological Record 2014).

- Before an importation or environmental detection occurs, IPV-only countries should re-evaluate their risk of WPV importation and consider ensuring access to monovalent OPV stocks. This will be particularly important as type 2 viruses are removed from tOPV with the proposed shift in OPV-using countries from tOPV to bOPV for routine immunization in April 2016 and eventually to no OPV.

- A sequential schedule using both OPV and IPV is ideal in areas where the risk of WPV introduction is considered high.

\section{References}

Anis E, Kopel E, Singer SR, Kalinger E, Moerman L, Moran-Gilad J, Sofer D, Manor Y, Shulman LM, Mendelson E, Gdalevich M, Lev B, Gamzu R, Grotto I (2013) Insidious reintroduction of wild poliovirus into Israel, 2013. Euro Surveill 18(38):2-6

Goldblum N, Gerichter CB, Tulchinsky TH, Melnick JL (1994) Poliomyelitis control in Israel, the West Bank and Gaza strip: changing strategies with the goal of eradication in an endemic area. Bull World Health Organ 72:783-796

Mangal TD, Aylward RB, Grassly NC (2013) The potential impact of routine immunization with inactivated poliovirus vaccine on wild-type or vaccine-derived poliovirus outbreaks in a posteradication setting. Am J Epidemiol 178:1579-1587. doi:10.1093/ aje/kwt203

Manor Y, Shulman LM, Kaliner E, Hindiyeh M, Ram D, Sofer D, Moran-Gilad J, Lev B, Grotto I, Gamzu R, Mendelson E (2014) Intensified environmental surveillance supporting the response to wild poliovirus type 1 silent circulation in Israel, 2013. Euro Surveill 19(7):1-10

Moturi EK, Porter KA, Wassilak SG et al (2014) Progress toward polio eradication-worldwide, 2013-2014. MMWR Morb Mortal Wkly Rep 63:468-472

Mundel T, Orenstein WA (2013) No country is safe without global eradication of poliomyelitis. N Engl J Med 369:2045-2046. doi:10.1056/NEJMe1311591

Slater P, Costin C, Yarrow A et al (1990) Poliomyelitis outbreak in Israel in 1988: a report with two commentaries. Lancet 335:1192-1195

Tulchinsky TH, Ramlawi A, Abdeen Z et al (2013) Polio lessons 2013: Israel, the West Bank, and Gaza. Lancet 382:1611-1612. doi:10.1016/S0140-6736(13)62331-4

Weekly Epidemiological Record (2014) Polio vaccines: WHO position paper January 2014. World Health Organization, Geneva

World Health Organization (2013) Polio Eradication and Endgame Strategic Plan 2013-2018. Global Polio Eradication Initiative. Available via GPEI. http://www.polioeradication.org/Portals/0/ Document/Resources/StrategyWork/PEESP_EN_US.pdf. Accessed 28 May 2015 